\title{
LA REACTIVIDAD CONSTITUCIONAL DEL SUJETO. UNA "PIEDRA DE ROSETTA" INTERDISCIPLINARIA
}

\author{
Pietro Magliozzi ${ }^{1}$
}

Resumen: Aprender a identificar de manera integral, mixta y dinámica la constitución de un sujeto significa, por un lado, ayudarlo a autoconocerse en su manera de reaccionar (por eso se habla de reactividad constitucional y no más de biotipos o rasgos), de desarrollarse (madurar o destruirse), de relacionarse y aceptar a los demás tal como son; por otro lado, sirve para personalizar diagnóstico, terapia y pronóstico.

La historia del constitucionalismo y un esquema sinóptico interdisciplinario (filosofía griega, teología, medicina hipocrática, galénica, homeopática, china, ayurvédica y holística, biología, embriología, bioquímica, fisiopatología, neurología, endocrinología, psicología y espiritualidad) muestra una precisa correspondencia y un denominador común sobre la base de la teoría constitucional embriológica que habla de endoblasto, mesoblasto y ectoblasto. Una coherencia que dura por más de 24 siglos en occidente y pasa también las barreras culturales (oriente y occidente) y paradigmáticas (biomedicina y homeopatía), no puede ser casual.

Palabras clave: interdisciplinaridad, endoblasta, mesoblasta, ectoblasta, constitución, personalización

\section{Subject's constitutional reactivitya. Interdisciplinary "rosetta stone"}

\begin{abstract}
To learn to identify in an integral, mixed and dynamic way the subject's constitution means, on one hand, to help him to a self-knowledgement of his reactive way (for this, we speak of constitutional reactivity and no more of biotipe or trait), to develop himself (to mature or to destroy), to join and accept the others like they are; on the other way, it serve to personalize diagnosis, therapy and prognosis.

The constitution history and a synoptic interdisciplinary diagram (Greek philosophy, theology, hippocratic, galenic, homeopatic, chinese, ayurvedic and holistic medicines, biology, embriology, biochemistry, physiopathology, neurology, endocrinology, psychology and spirituality) shows a precise link and a common denominator according to an embriologic constitutional theory, that speaks about endoblast, mesoblast and ectoblast. A coherence thas lasts for more than 24 centuries in occident and goes through even the cultural borders (eastern and western world) and paradigmatic logics (biomedicine and homeopaty) cannot be casual.
\end{abstract}

Key words: interdisciplinarity, endoblast, mesoblast, ectoblast, constitution, personalization

\section{A reatividade constitucional do sujeito. Uma "pedra de roseta" interdisciplinar}

Resumo: Aprender a identificar de maneira integral, mista e dinâmica a constituição de um sujeito significa, por um lado, ajudá-lo a se autoconhecer na sua maneira de reagir (por isso se fala de reatividade constitucional e não mais de biotipos ou traços), de desenvolver-se (amadurecer ou destruir-se), de relacionar-se e aceitar os demais taisl como são; por outro lado, serve para personalizar diagnóstico, terapia e prognóstico.

A história do constitucionalismo e um esquema sinóptico interdisciplinar (filosofia grega, teologia, medicina hipocrática, galênica, homeopática, chinesa, ayurvédica e holística, biologia, embriologia, bioquímica, fisiopatologia, neurologia, endocrinologia, psicologia e espiritualidade) mostra uma precisa correspondência e um denominador comum sobre a base da teoria constitucional embriológica que fala de endoblasto, mesoblasto e ectoblasto. Uma coerência que dura por mais de 24 séculos no ocidente e ultrapassa também as barreiras culturais (oriente e ocidente) e paradigmáticas (biomedicina e homeopatia), não pode ser casual.

Palavras-chave: interdisciplinaridade, endoblasto, mesoblasto, ectoblasto, constituição, personalização

\footnotetext{
${ }^{1}$ Programa de Estudios Médicos Humanísticos, Facultad de Medicina, Pontificia Universidad Católica de Chile, Chile Correspondencia: pmaglioz@med.puc.cl
} 


\section{Introducción}

La Piedra de Rosetta es un fragmento de una antigua estela egipcia de un decreto publicado en Menfis en 196 a. C., en nombre del faraón Ptolomeo V. El decreto aparece en tres escrituras distintas: egipcio, escritura demótica y griego antiguo. Gracias a que presenta esencialmente el mismo contenido, esta piedra facilitó la clave para entender los jeroglíficos egipcios. Hoy el principal problema interdisciplinario es cómo hacer dialogar las varias ciencias y disciplinas, cómo encontrar una clave para comparar las distintas disciplinas humanas, científicas duras y teológicas; o cuantitativas y cualitativas. El artículo quiere solo, volver a presentar en términos actuales una antigua clave de lectura interdisciplinaria: el constitucionalismo.

El temperamento, según Mario Fedeli, médico y psicólogo(1), es la parte más estable del propio yo, porque está vinculada al patrimonio genético de cada uno. También Arnold Buss y Robert Plomin, psicólogos(2:311-314), definieron en 1984 como temperamento la parte genética de la personalidad caracterizada por tres dimensiones: a) rasgos heredados; b) rasgos estables desde la infancia y que perduran en la madurez; c) rasgos que tienen o han tenido un valor adaptativo en la evolución de la raza humana. Estudios sobre gemelos monocigotos contra gemelos dicigotos sirvieron para diferenciar el temperamento genético del carácter de origen psico-socio-educativo y de la personalidad. El carácter y la personalidad(2) son la parte variable de la persona. El temperamento, es la "forma" (física y mental) que la persona tiene, es lo que uno es. Carácter y personalidad, son lo en que uno se vuelve o quiere volverse.

La personalidad es el efecto de la interacción entre factores biológicos (determinados genéticamente), situaciones ambientales (psico-socio-pedagógicas) y elecciones libres (éticas, espirituales) del sujeto. La personalidad se desarrolla (en positivo o en negativo) por acciones externas (sociales, culturales, educativas) e internas (la voluntad y libertad del sujeto), pero siempre en un rango de posibilidades definidas por el temperamento propio.

La combinación de temperamento, carácter y personalidad es definida como la constitución de un sujeto o aptitud reaccional (morfo-fisio-psicológica) como sano y enfermo. La constitución es, por lo tanto, lo que uno es reactivamente; se ve durante toda la existencia, en todas las manifestaciones del sujeto, en todos sus procesos vitales y patológicos. La constitución es para Stanley Keleman(3), terapeuta constitucional, la forma instintiva fundamental para estar en el mundo de una determinada manera.

La constitución del sujeto denota cómo esa persona reacciona a las experiencias externas, a los demás y a su mundo interior. Conocer la constitución es conocer el "lenguaje" que habla el propio organismo, la mente y el espíritu, o, mejor dicho, el todo de la persona. Se trata, en síntesis, de la integralidad personalizada del sujeto.

En el presente artículo se usará la teoría embriológica-constitucional(4-10) que identifica tres reactividades constitucionales integrales (con características estructurales, funcionales y psicológicas). En particular será adoptada la terminología de Martiny, Castellino y Sheldom, que hablan de biotipos endoblasta, mesoblasta y ectoblasta. El término "biotipo" no denota una "naturaleza", sino una "reactividad" (una manera de reaccionar), una función, un oficio, un proceso de acciones hacia una dirección.

Por tanto, decir, "el sujeto tiene el biotipo X", no significa encasillarlo: "pertenece al grupo X, es X y no puede cambiar", como si definir el biotipo fuera un juicio sobre el "ser" del individuo, sino: "esta persona está en este proceso, está funcionando en esta dirección, está reaccionando así y se dirige orgánica, mental y espiritualmente hacia este objetivo, su proyecto personal biológico y biográfico tiene este rumbo". De este modo se respeta mucho más la libertad del sujeto y es más fácil ayudarlo a cumplir su proyecto personal identificando sus fines y sus recursos interiores.

¿Por qué es importante conocer la propia reactividad constitucional? Cuatro son los objetivos de este tipo de conocimiento(1):

- Conocer las propias necesidades, tendencias y expectativas (autoconocimiento).

- Conocer las propias potencialidades y posibilidades (desarrollo y autorrealización). 
- Conocer a los demás y aceptarlos como son (mejorar las relaciones).

- Mejorar la relación del agente de la salud con el paciente (favorecer la calidad y disminuir demandas en ambiente sanitario)(11).

Conrado Hock profundiza las tres ventajas del conocimiento biotipológico en su texto(12).

Autoconocerse, significa apreciarse y aceptarse, saber que si hay aspectos vulnerables y límites, también hay aspectos positivos y fuertes, y con estos se puede mejorar la propia autoestima y la imagen de sí. Cuando uno se autoconoce no depende más del juicio, de la crítica ni del insulto de los demás. La persona sabe que las virtudes no son siempre fruto de su fuerza de voluntad, sino de su reacción tipológica, y que los defectos están muchas veces también vinculados a su tipología².

Autorrealizarse, significa saber como autoeducarse para controlar los aspectos destructivos de sí y fortalecer los constructivos. O sea, solo con este conocimiento es posible saber en qué trabajar, qué

${ }^{2}$ Por ejemplo, la fuerza de voluntad y energía del colérico; la facilidad en tratar con caracteres difíciles del sanguíneo; la profundidad del alma, el amor a la soledad y a la oración del melancólico; la amabilidad y la calma del flemático, son virtudes que no implican esfuerzo, sino son tipológicas. Por lo mismo, el ser duro, impetuoso, obstinado del colérico; el ser tímido, indeciso del melancólico; el ser inconstante y superficial del sanguíneo; el ser perezoso del flemático, no son vicios por mala voluntad, sino son tipológicos. cultivar como cualidades, dónde y cómo luchar para mejorar.

Mejorar las relaciones es importante, porque solo conociendo al otro en su constitución, podemos aceptarlo y comprenderlo (sentir que las diferencias sirven para completarse y ayudarse y no para rechazarse), tratarlo con justicia (y no por prejuicios sociales o culturales), se puede educarlo (sacar de él lo mejor que tiene) y se puede tener más paciencia con él.

El conocimiento constitucional del enfermo permite al agente de salud, si quiere integrar el modelo personal de alianza, que considera al paciente como persona digna y autónoma, humanizar la relación sanitaria. Los modelos de relación tecnológico, consumista, liberal o contractual, paternalista estatal no están pensados para respetar la dignidad de la persona ni para ver al paciente o al agente de la salud como sujetos, sino como objetos y medios de una institución de producción y fomentan el conflicto y la reivindicación de cada vez más derechos. En cambio, el modelo de alianza (una confianza en una conciencia), posible solo en una relación personalizada, integral y de crecimiento, permite un clima de búsqueda y discernimiento armónico. Los límites de tal modelo de conocimiento constitucional serían la necesidad de una competencia específica y de dedicar más tiempo al paciente; por tanto, sería complejo
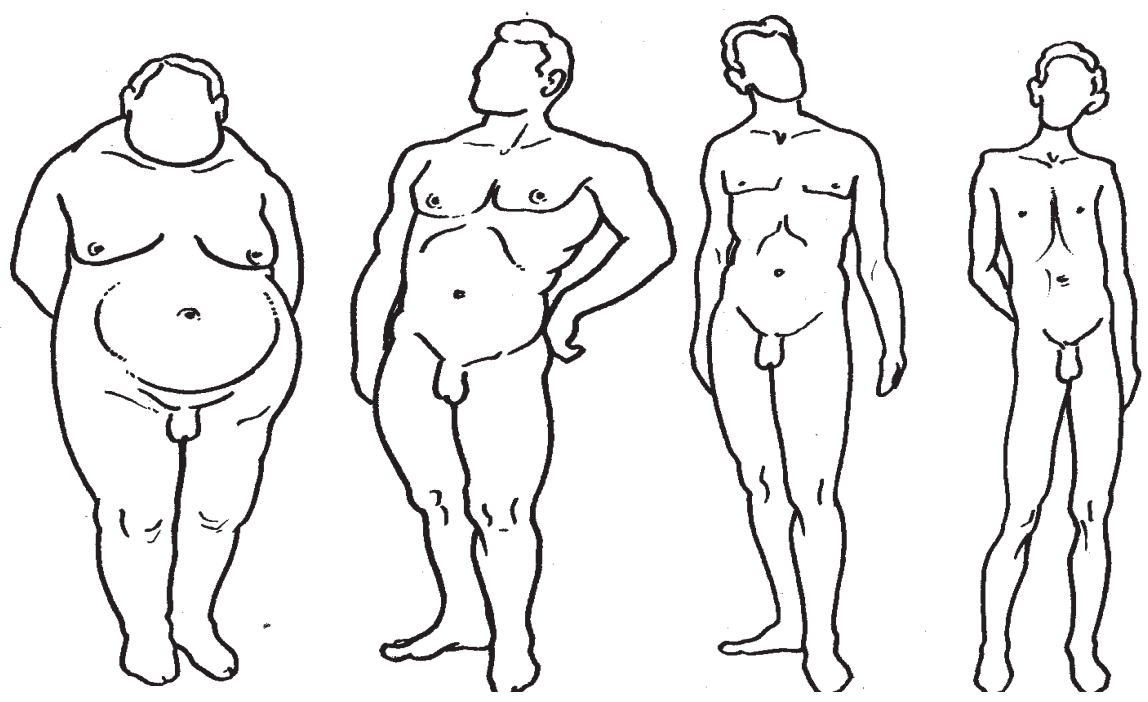

Los cuatro biotipos constitucionales, sintetizables en tres (por Antonio Santini)(14) 
aplicarlo sin un aumento de costo de la sanidad a la totalidad de los pacientes institucionalizados, a menos que se constituya un equipo de trabajo integral acerca del paciente.

La historia de la teoría embriológica-constitucio$\operatorname{nal}(9,12,13)$

Toda la biotipología constitucional, desde la escuela hipocrática hasta hoy, se ha ido perfeccionando paulatinamente y entendiendo cada vez más en profundidad, en un esquema biotipológico único, que forma la teoría embriológica constitucional (tabla 1).

\section{Semiología embriológica(15)}

Los términos que se usarán en el texto para identificar las distintas reactividades (endoblasta, mesoblasta y ectoblasta), tomados por una genial intuición de Pietro Castellino (1927) y luego desarrollados por Marcel Martiny (1948) y William H. Sheldom (+1977), originan de: endoderma, mesoderma y ectoderma, que son las tres capas celulares (interna, media y externa) que se desarrollan del blastocisto en el disco embrionario desde 15 días de la concepción (tabla 2).

Endoblasta, Mesoblasta y Ectoblasta son las tres reactividades biotipológicas (la morfológica, la funcional y la mental) en las cuales predomina el desarrollo de una de las tres capas embrionarias.

Si en el embrión se desarrolla más la capa endodérmica, el individuo evolucionará morfológicamente como brevilíneo (bajo y redondo), débil (poco desarrollo muscular), megalospláncnico (con gran abdomen), fisiológicamente bajo el dominio vagal (asimilativo, tendencia a acumular grasas más que a consumir energía, bioquímicamente alcalótico).

Si en el embrión se desarrolla más la capa mesodérmica, el individuo evolucionará morfológicamente como sujeto robusto, hombros anchos, cadera estrecha, fisiológicamente estará bajo el dominio del ortosimpático (oxidativo, consumiendo más energía de la que asimila, bioquímicamente acidósico).

Si en el embrión se desarrolla más la capa ectodérmica el individuo en cambio, evolucionará mor- fológicamente como esbelto, débil, microspláncnico (abdomen reducido), etc.

El esquema histórico es el que muestra la tabla $3(1,10,16)$.

En este esquema sinóptico se demuestra como la historia de las biotipologías humanas sigue un rumbo unitario - a pesar de los muchos términos utilizados-y se muestran las correspondencias y el denominador común en un nivel embriológico y fisiopatológico que fundamenta los tres o cuatro biotipos constitucionales en varias tradiciones culturales y paradigmas médicos. Una homogeneidad y coherencia que dura por más de 24 siglos y pasa también las barreras culturales y paradigmáticas (es común a la medicina convencional y homeopática, a la occidental y oriental), no puede ser casual.

Llama también la atención que estas numerosas teorías constitucionales empiezan con un planteamiento integral (en la antigua escuela griega y en el medioevo), para pasar a un enfoque puramente estructural o morfológico (escuela francesa siglos XVIII-XIX) o puramente funcional (escuela alemana siglos XIX-XX) o morfo-funcional (escuela italiana siglos XIX-XX), para regresar a una perspectiva más integral (morfo-funcional-psicológica escuela estadounidense y rusa, homeopática y oriental) en el siglo XX-XXI.

Se partió de una integralidad sin cientificidad (medicinas china, ayurveda y griega antiguas), para pasar a una cientificidad sin integralidad (escuelas médicas francesa, alemana e italiana del siglo 19), para llegar en el siglo XX y XXI, gracias a la interdisciplinaridad, a una medicina constitucional, en la cual integralidad y cientificidad conviven. Todo esto podrá ayudar a construir una medicina antropológica y humanizada para la persona.

\section{Los biotipos embriológicos constitucionales hoy}

Hablar de biotipos constitucionales no significa entrar en un determinismo o mecanicismo (biotipo $\mathrm{x}=$ efecto $\mathrm{x}$ ) que impide modificaciones, sino contar con una pista con la cual la persona puede crecer o entramparse, avanzar o retroceder según 
Tabla 1: Modelo embriológico - constitucional

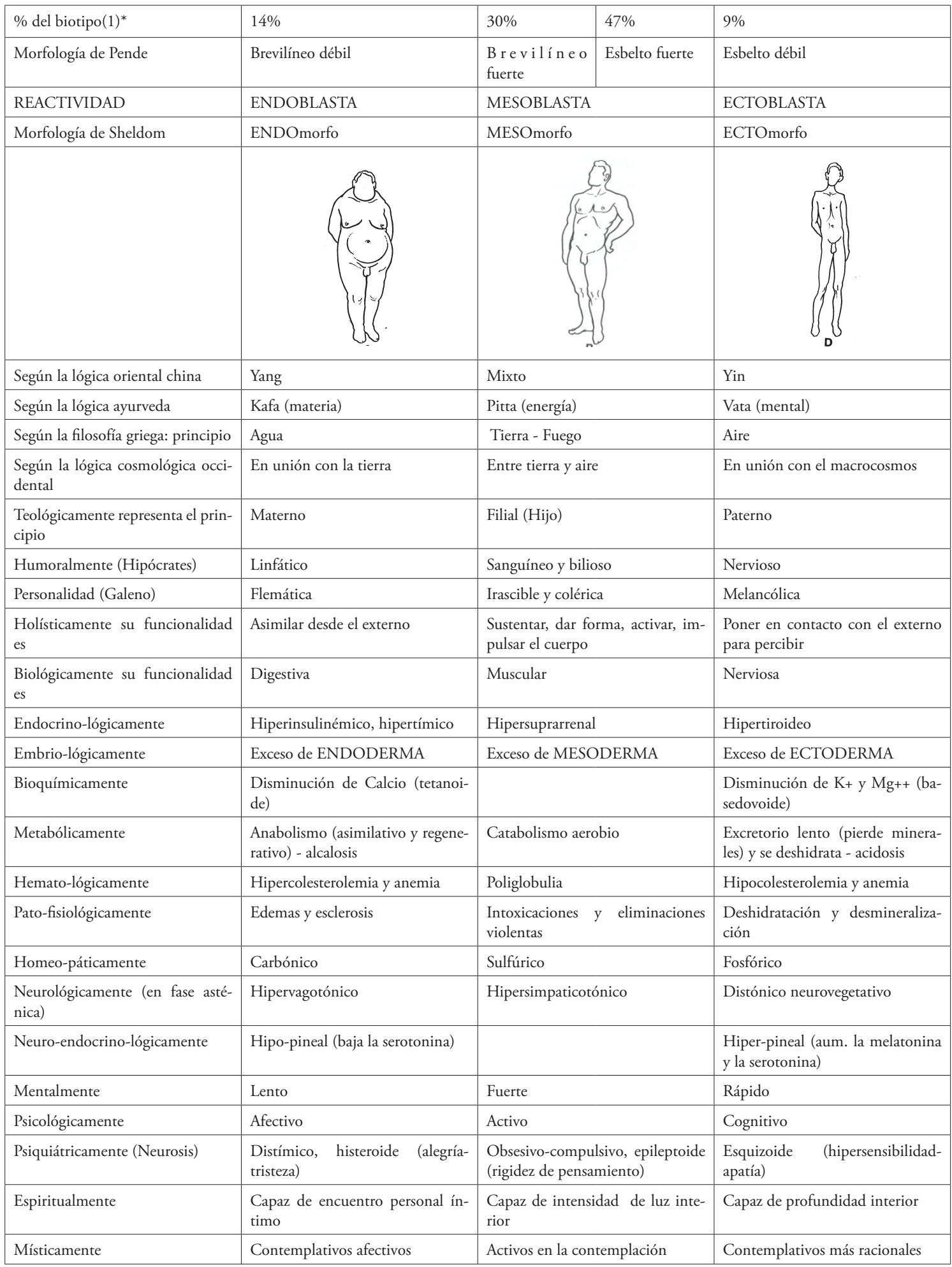

* Los datos son de C. Sigaud, por lo tanto, de la Francia del siglo XIX. 
Tabla 2.

\begin{tabular}{|c|c|c|c|}
\hline $\begin{array}{l}\text { Desde la } 4^{\circ} \text { hasta la } 8^{\circ} \text { semana de } \\
\text { desarrollo }\end{array}$ & Del Endodermo se origina: & Del Mesodermo se origina: & Del Ectodermo se origina: \\
\hline $\begin{array}{l}\text { ÓRGANOS vinculados a la capa } \\
\text { embrionaria }\end{array}$ & $\begin{array}{l}\text { El revestimiento mucoso del } \\
\text { aparato respiratorio, digestivo y } \\
\text { urinario (vejiga y uretra) y otros } \\
\text { órganos como el hígado, páncreas, } \\
\text { glándulas paratiroides, tiroides, } \\
\text { amígdalas, timo, cavidad timpá- } \\
\text { nica. }\end{array}$ & $\begin{array}{l}\text { El tejido conectivo, el sistema pi- } \\
\text { lífero, el sistema músculo-esquelé- } \\
\text { tico, cardiovascular y linfático, la } \\
\text { sangre, más los rińones y suprarre- } \\
\text { nales, bazo, el sistema reproductor } \\
\text { (gónadas y ductos) y las serosas } \\
\text { (pleura, pericardio y peritoneo) }\end{array}$ & $\begin{array}{l}\text { El sistema nervioso (central y } \\
\text { periférico) y cutáneo (pelo de la } \\
\text { cabeza, uñas, glándulas cutáneas), } \\
\text { los órganos de los sentidos (ojos, } \\
\text { oídos), mucosas de boca y ano, } \\
\text { glándula mamaria, hipófisis, es- } \\
\text { malte de los dientes. }\end{array}$ \\
\hline $\begin{array}{l}\text { SIGNIFICADO evolutivo de la } \\
\text { capa embrionaria }\end{array}$ & $\begin{array}{l}\text { Todo lo que sirve al sujeto para } \\
\text { asimilar (recibir) desde el externo } \\
\text { (oxigeno, agua, alimentos) y para } \\
\text { eliminar toxinas. }\end{array}$ & $\begin{array}{l}\text { Todo lo que da estructura y forma } \\
\text { al cuerpo. Lo que permite al cuer- } \\
\text { po interno ser dinámico (moverse } \\
\text { en el espacio). }\end{array}$ & $\begin{array}{l}\text { Todo lo que pone en contacto } \\
\text { con el mundo externo y sirve para } \\
\text { sentir (percibir) esta dimensión } \\
\text { externa. }\end{array}$ \\
\hline
\end{tabular}

el uso que hace de sus "recursos" constitucionales. En un cierto sentido, los recursos constitucionales son los talentos recibidos para invertirlos y con los cuales uno tiene que con-vivir en este mundo. Keleman usa la siguiente expresión: constitución es el "marco" para el diálogo interno (y externo) del sujeto, es decir:

- Un marco inflado, blando o débil funcionalmente (endoblasta): biotipo lleno.

- Un marco denso o rígido (mesoblasta): biotipo uno.

- Un marco poroso, frágil o débil estructuralmente (ectoblasta): biotipo en que falta algo.

El sujeto es una mezcla de los tres marcos reactivos y, a lo largo de su vida, desarrolla o atrofia el uno o el otro en un dinamismo que es absolutamente personal. La constitución morfológica, funcional y psicoespiritual del sujeto, en efecto, está en continua evolución, sobre todo en su dimensión psicoespiritual (por esto la constitución es siempre mixta y dinámica).

La reactividad es mixta (en el $90 \%$ de los sujetos) según el contexto externo. Ejemplos:

- El sujeto es endoblasto y cálido en las relaciones interpersonales y ectoblasto y frío en el escribir o viceversa.

- El sujeto es meso y agresivo con algunas personas de la familia y ectoblasto y tímido con otras personas fuera de la casa.

- El sujeto es extravertido y alegre en su trabajo y aislado e introvertido en casa.

- Un sacerdote es depresivo si es puesto con la responsabilidad de formar a estudiantes y es heroico como ecónomo y capellán (B. Henrique Rebuschini).

- Es infeliz como comerciante y feliz como consagrado (S. Francisco de Asis).

También la reactividad tiene un compuesto mixto en un nivel morfológico, otro en un nivel funcional, otro en un nivel mental. Stanley Keleman hipotetiza, sin profundizarlo, una sobreposición, temporalmente sucesiva, de capas biotipológicas, tipo "matrioskas".

\section{La reactividad es dinámica. Ejemplos:}

- En el embarazo, la mujer pasa de una reactividad ecto a una meso, porque alguna causa fisiológica, psicológica o patológica (a carácter mesoblastizante) empezó a actuar en ella.

- Puede pasar de ecto a endo (engorda después de un embarazo), porque un carácter endoblastizante actuó en ella.

- Un meso-ectoblasto va adelgazando si el biotipo mesoblasto tiene estilo neurótico que lo empuja hacia el ecto.

- Al contrario, un ectoblasto puede volverse un mesoblasto si encuentra estímulos que lo fortalecen.

Con la teoría constitucional se puede explicar el 
Tabla 3

Autores constitucionalistas y teorías constitucionales

\begin{tabular}{|l|l|l|l|l|}
\hline & & \\
\hline & & \multicolumn{2}{|l|}{} \\
\hline Hipócrates & 1 & 2 & 3 & \\
\hline Platón & Linfático & Sanguíneo & Bilioso & Atrabiliare \\
\hline San Alberto Magno & Thymós (emociones) & Epithymía (deseos) & Nús (inteligencia) \\
\hline Lazaro Riverio & Sońoliento, perezoso & Alegre, ingenioso & Inestable, vehemente, & Insomne, solitario \\
\hline Alquimistas & Flemático & Sanguíneo & Colérico & Melancólico \\
\hline G.B. Morgagni & Mercurial & Sulfúrico & Sulfúrico & Salino \\
\hline
\end{tabular}

\section{Escuela constitucional francesa (morfológica)}

\begin{tabular}{|l|l|l|l|l|}
\hline R. Allendy & Átono-plástico & Tono-plástico & Tono-aplástico & Átono-aplástico \\
\hline C. Sigaud & Digestivo & Respiratorio & Muscular & Cerebral \\
\hline P. Castellino M. Martiny & Endoblasta & Mesoblasta & Cordoblasta & Ectoblasta \\
\hline L. Corman & $\begin{array}{l}\text { Expansión (dilatado) pa- } \\
\text { siva }\end{array}$ & Expansión activa & $\begin{array}{l}\text { Retracción (contraído) } \\
\text { activa }\end{array}$ & Retracción pasiva \\
\hline
\end{tabular}

Escuela constitucional alemana (funcional)

\begin{tabular}{|l|l|l|l|l|}
\hline G. Stahl & \multicolumn{2}{|l|}{ Túrgido-hidrofilos } & \multicolumn{2}{l|}{ Secos-hidrófobos } \\
\hline E. Kretschmer & Pícnico ciclotímico & Atlético ideas lentas & Displástico epiléptico & $\begin{array}{l}\text { Leptosómico esquizotí- } \\
\text { mico }\end{array}$ \\
\hline L. Borchardt & Asténico & Esténico & Esténico & Asténico \\
\hline W. Jaensch & Tetanoide o T & Basedovoide B & Basedovoide B & Tetanoide o T \\
\hline
\end{tabular}

Escuela constitucional estadounidense y rusa

\begin{tabular}{|l|l|l|l|l|}
\hline W.H. Sheldom & Viscerotónico endomorfo & Somatotónico sanguíneo & Somatotónico mesomorfo & Cerebrotónico ectomorfo \\
\hline I.Pavlov & Flemático tranquilo & Sanguíneo vivaz & Colérico impetuoso & Melancólico débil \\
\hline
\end{tabular}

Escuela constitucional italiana (morfofuncional)

\begin{tabular}{|l|l|l|l|l|}
\hline G. Viola & $\begin{array}{l}\text { Brevilíneo megalosplánc- } \\
\text { nico }\end{array}$ & Normal & Esbelto microspláncnico \\
\hline N. Pende & Braquitipo asténico & Braquitipo esténico & Esbelto esténico & Esbelto asténico \\
\hline N. Pende & $\begin{array}{l}\text { Hipotiroideo hipervago- } \\
\text { tónico }\end{array}$ & $\begin{array}{l}\text { Hipersuprarrenálico hiper- } \\
\text { simpático }\end{array}$ & $\begin{array}{l}\text { Hipertiroideo hipersim- } \\
\text { pático }\end{array}$ & $\begin{array}{l}\text { Hiposuprarrenálico distó- } \\
\text { nico }\end{array}$ \\
\hline
\end{tabular}


Medicina homeopática constitucional

\begin{tabular}{|l|l|l|l|l|}
\hline A.Nebel & Carbo-cálcico & \multicolumn{2}{|l|}{ Flúoro-cálcico } & \multicolumn{2}{|l|}{ Flúoro-cálcico } & Fósforo-cálcico \\
\hline L. Vannier & Carbónico & Fluorico & Fosfórico \\
\hline H. Bernard & Carbónico & Sulfur gordo & Sulfur delgado & Fosfórico \\
\hline R. Zissü* & Asténico (débil) & Esténico (fuerte) & Asténico \\
\hline E. von Grauvogl & Hidrogenoide & Carbonitrógeno & Oxigenoide \\
\hline $\begin{array}{l}\text { Evolución de la patología } \\
\text { hacia: }\end{array}$ & $\begin{array}{l}\text { Sicosis gorda o tubercolis- } \\
\text { mo floreciente }\end{array}$ & Psorismo & Luetismo & $\begin{array}{l}\text { Sicosis delgada tuberculi- } \\
\text { nismo delgado }\end{array}$ \\
\hline $\begin{array}{l}\text { Fisiopatologia de la evo- } \\
\text { lución: }\end{array}$ & Esclerosis, edema & $\begin{array}{l}\text { Eliminación externa de } \\
\text { toxinas }\end{array}$ & Destrucción de tejidos & $\begin{array}{l}\text { Deshidratación Desmine- } \\
\text { ralización }\end{array}$ \\
\hline
\end{tabular}

Medicinas constitucionales de Oriente

\begin{tabular}{|l|l|l|l|}
\hline Medicina china & $\begin{array}{l}\text { YIN - femenino Chakras 1 y 2 } \\
\text { (bajo abdomen) }\end{array}$ & $\begin{array}{l}\text { MIXTO Chakras 3 y 4 (alto abdo- } \\
\text { men y tórax) }\end{array}$ & $\begin{array}{l}\text { YANG - masculino Chakra 5, 6 y } \\
7 \text { (cuello y cabeza) }\end{array}$ \\
\hline Medicina ayurveda & CAPHA Estructura & PITTA Energía & VATA Mental \\
\hline Místicos del islam - sufos & Biotipo CORAZÓN & Biotipo VISCERAL & Biotipo CABEZA \\
\hline Enneatipos & $2,7,9$ & $1,3,8$ & $4,5,6$ \\
\hline Bioenergética W. Reich, A. Lowen & ORAL & $\begin{array}{l}\text { MASOQUISTA; PSICOPÁTI- } \\
\text { CO ESUIZOIDE }\end{array}$ \\
\hline
\end{tabular}

* Las constituciones son, para R. Zissú: Carbónico-endoblasta, Sulfúrico-mesoblasta, Fosfórico-ectoblasta. Los caracteres son: Linfático, Sanguíneo, Bilioso, Nervoso.

pasado del sujeto. Se puede interpretar la evolución de las enfermedades de un sujeto y se puede llegar a entender el pronóstico de sus enfermedades y ayudar a buscar tratamientos personalizados.

Ejemplos de reactividad constitucional(3)

Cada ser humano vive según una constitución prioritaria:

\begin{tabular}{|l|l|l|l|}
\hline Reactividad & Endoblasta & Mesoblasta & Ectoblasta \\
\hline Orgánica & Digestiva & Muscular & Nerviosa \\
\hline Mental & Afectiva & Activa & Cognitiva \\
\hline Espiritual & $\begin{array}{l}\text { Que encuentra } \\
\text { íntimamente } \\
\text { al otro }\end{array}$ & $\begin{array}{l}\text { Que busca la } \\
\text { intensidad de } \\
\text { luz interior }\end{array}$ & $\begin{array}{l}\text { Que busca su } \\
\text { profundidad } \\
\text { interior }\end{array}$ \\
\hline
\end{tabular}

Ejemplos:

1. Frente al estrés, el biotipo endoblasto colapsa (es como si se congelara), el mesoblasto ataca, el ectoblasto se pone nervioso.

2. Frente al tiempo, el endoblasto lo vive como periodos cíclicos sin límites, necesita de mucho tiempo para hacer las cosas (vive con cal$\mathrm{ma}$ ); el mesoblasto como secuencias de actos en forma lineal (como desafío), y el ectoblasto lo percibe como serie de instantáneas de la realidad, proyectado al futuro (con ansiedad).
3. Frente al amor, el endoblasto pide sentirse querido y conectado (expresa: "sabes que te quiero por el hecho de estar contigo", "te acojo en mî"), entra en profunda empatía y compasión con el otro, espera que se le quiera, cuidándole. El mesoblasto vive el amor como hacer (dice: "sabes que te quiero por lo que hago por ti"), a veces es compasivo y empático, pero generalmente carece de ternura, busca el amor como aprobación y respuesta de estima a su acción; la falta de atención le desanima. El ectoblasto vive el amor dando atención, entregándose, pero a distancia (sin intimidad ni cercanía), se autosacrifica, pero sin dirección fija, más compasivo que empático, quiere compartir sus intereses con los demás.

4. Frente a la experiencia de las cosas, el endoblasto se sintoniza, entra en armonía con las cosas, las contiene; el mesoblasto trabaja con las cosas, siendo activo y parte de estas para transformarlas. El ectoblasto calla y está presente frente a las cosas, analizándolas a través de la alerta.

5. Frente a la experiencia del cambio, el endoblasto se pregunta: "¿Debo esperar para digerirlo?”; el mesoblasto “¿Debo empezar 
a actuar inmediatamente?”; el ectoblasto “¿Debo percibir y reunir informaciones sobre la experiencia?”. La reacción del endoblasto es absorción; del mesoblasto, actividad; del ectoblasto, control mental.

En síntesis, la personalidad del endoblasto es cálida y extrovertida, con pasajes de la euforia a la tristeza. Se caracteriza por su lentitud de reacción. La personalidad del mesoblasto presenta actividad incesante: el entusiasmo y el combate o confrontación, como modo de ser. La personalidad del ectoblasto está vinculada con la superficie sensitiva (piel y sistema nervioso) con la cual conoce al mundo, recibe muchos estímulos y luego los elabora en su mente, tomando contacto con cosas y personas esporádicamente, estableciendo relaciones y abandonándolas; se caracteriza por la no confrontación y el exceso de contacto lo agota.

Un esquema que simplifica y sintetiza la personalidad de las tres reactividades constitucionales es el siguiente.

\section{Implicaciones éticas del constitucionalismo}

Arnoldo de Villanova escribía en el siglo XII que la medicina es ciencia global que no solo es un medio para conservar la salud del cuerpo, sino también para "perfeccionar la propia vida", para "la formación del espíritu". Pudiendo ampliar en el siglo XXI la vocación del agente de salud (médico incluido) y el horizonte de las ciencias sanitarias (las duras y las humanidades médicas) a la dimensión constitucional del enfermo, es posible enriquecer tanto la ética principialista americana como la personalista católica.

El principialismo, con el conocimiento constitucional, puede ayudar al paciente a solucionar algunos casos de conflicto entre la autonomía (el bien para mí) y la beneficencia (el bien en sí). El personalismo puede también, con este conocimiento, favorecer el proceso de personalización de la prevención, diagnóstico, terapia y pronóstico, cuya necesidad es frecuentemente expresada en teoría, pero difícil en su actuación concreta. Además, cuando el conocimiento constitucional empieza a ser parte de la medicina antropológica, como la de Viktor von Weizsäcker en Alemania o la de Nicola Pende en Italia, las ventajas son también en el plano de una cura integral e interdisciplinaria, de una relación de alianza entre funcionarios, pacientes y familiares, de comprensión del dinamismo biográfico del paciente.

Se trata, sin embargo, de hipótesis de trabajo que necesitan ser puestas en marcha e institucionalizadas para averiguar sus resultados, y las experiencias, hasta el momento, son pocas y poco estudiadas(17:117-141).

\section{Conclusión}

Cada reactividad constitucional inserta en una persona se mueve en dos direcciones: hacia el Mal (el desequilibrio, la desestructuración de sí mismo) o hacia el Bien (la plenitud de su vida, la armonía e integración de las varias dimensiones de su ser).

La ayuda constitucional interdisciplinaria consiste en dar facilidad de concientizar este proceso y saber cómo moverse hacia el propio Bien, la única manera de transformar las necesidades (los negativos) en dones (positivos) y vivir positivamente los "talentos".

Este movimiento entre los dos polos es algo semejante al equilibrio del parasimpático y ortosimpático, del yin y yang. Si el sujeto se dirige al polo "Bien" solo con su mente (con un esfuerzo de concentración o de visualización), solo con su emoción (con una experiencia emocional fuerte, un rito), solo con sus deseos o voluntad o gestos, luego hay un efecto rebound y el sujeto regresa hacia el Mal. Lo mismo pasa si el objetivo es llegar a un "punto estático" (un comportamiento, una actitud, una virtud) y luego no poder crecer más.

El movimiento constitucional es eficaz si es continuo hacia el Bien en una actitud de dinamismo espiritual hacia una imagen dinámica, es decir, un camino de espiritualidad.

Así como a través de la "Piedra de Rosetta", gracias a la correspondencia de tres contenidos en tres idiomas distintos, conociendo un idioma (el griego), se pudo traducir e interpretar a otro desconocido (egipcio), así también, a través de tablas sinópticas como la presentada, se podrán interpretar de manera cada vez más integral e interdis- 
La reactividad constitucional del sujeto. Una "piedra de rosetta" interdisciplinaria - Pietro Magliozzi

ciplinaria fenómenos humanos (contenidos) que pero no interdisciplinaria. hoy son analizados solo de manera unidisciplinaria (un idioma) o, al máximo, pluridisciplinaria,

Referencias

1. Fedeli M. Temperamenti, caratteri, personalitá. Profilo medico e psicológico. Cinisello Balsamo (MI): San Paolo; 1997.

2. Engler B. Introducción a las teorias de la personalidad. Santiago de Chile: Mc Graw-Hill; 2002.

3. Keleman S. Love, a somatic view. Berkeley: Center press; 1994.

4. Christian P. Medicina antropológica. Santiago de Chile: Universitaria; 1997.

5. Magliozzi P. Psicofarmacologia sintomatica e omeopatia clinica nelle nevrosi, questioni etiche, antropologiche e pastorali, Dissertatio ad Lauream. Roma: Pontificia Facoltá Teologia Teresianum, Camillianum; 1999.

6. Pende N. La scienza moderna della persona umana, biologia, psicologia, tipologia normale e patologica, applicazioni mediche, pedagogiche e sociologiche. Milano: Garzanti; 1953.

7. Pende N. Trattato di biotipologia umana individuale e sociale con applicazioni alla medicina preventiva, al clima, alla politica biologica, alla sociologia. Milano: Francesco Vallardi; 1939.

8. Sankaran R. Schemi diagnostico-terapeutici. Padova: Salus Infirmorum; 2005.

9. Santini A. Omeopatia costituzionale. Tivoli (RM): Ismo, OTI, 1996.

10. Zissú R. Matiere medicale homeopathique constitucionelle. Etudes physio-patologique, étiologique, diathésique et clinique des remédes. France: Boiron; 1998.

11. Rodríguez Guerro A. Relación médico-paciente en algunos campos clínicos de la UC. Roma: IFPRESS; 2009.

12. Hock C. Los cuatro temperamentos. Su influencia en la formación y educación de la persona. México DF: Apóstoles de la Palabra; 2008.

13. Correnti V. Costituzione, en Enciclopedia Medica italiana, II. Firenze: USES, Ed. Scientifiche, 1985.

14. Castellino P. Dottrina dell'antagonismo e del sinergismo sull'equilibrio neurovegetativo. Bologna: Cappelli; 1929.

15. Langman J. Embriologia medica, Padova: Piccin; 1978.

16. Button N. Il codice umano. Un nuovo metodo per le tipologie costituzionali, Petah Tikva (Israel): Publ. Arti Grafiche Pergine Valsugana (TN); 2009.

17. Lolas F. La perspectiva psicosomática en medicina. Ensayos de aproximación. Santiago de Chile: Universitaria; 1995.

Recibido: 22 de agosto de 2012

Aceptado: 13 de noviembre de 2012 